\title{
MSLQ-UY, validación con estudiantes universitarios uruguayos
}

\section{MSLQ-UY, validation with Uruguayan university students}

\author{
Karina Curione * 1, Virginia Gründler ${ }^{2}$, Lucía Píriz ${ }^{3}$, Juan Antonio Huertas ${ }^{4}$ \\ 1 - Instituto de Fundamentos y Métodos en Psicología, Facultad de Psicología, Universidad de la República, Uruguay. \\ 2 - Administración Nacional de Educación Pública - Consejo de Educación Secundaria, Uruguay. \\ 3 - Facultad de Psicología, Universidad de la República, Uruguay. \\ 4 - Facultad de Psicología, Universidad Autónoma de Madrid, España.
}

Introducción
Método
Resultados
Discusión
Conclusiones
Referencias

Recibido: 23/04/2017 Revisado: 17/05/2017 Aceptado: 29/05/2017

\section{Resumen}

Se presenta la traducción, adaptación y validación al español de la versión abreviada del Motivated Strategies for Learning Questionnaire (MSLQ), desarrollado por Pintrich y De Groot (1990) para población uruguaya universitaria. El MSLQ fue construido desde el marco socio-cognitivo de la motivación y la autorregulación. El instrumento contiene 44 ítems que se responden con una escala tipo Likert de 1 a 7 y se divide en dos bloques denominados motivación y estrategias de aprendizaje autorregulado (EAA). La muestra incluyó 318 estudiantes de la Facultad de Psicología de la Universidad de la República (Uruguay). Los resultados evidencian que el bloque de motivación $(\alpha=.76)$ y el de estrategias de aprendizaje autorregulado $(\alpha=.75)$ poseen una consistencia interna aceptable para ser utilizados con fines de investigación. El análisis factorial exploratorio permitió confirmar la estructura original de cinco factores, aunque se plantea una reorganización del bloque estrategias de aprendizaje autorregulado.

Palabras clave: $M S L Q$, validación, motivación, aprendizaje autorregulado, Psicología

\begin{abstract}
The current study translates, adapts, and validates for Uruguayan university students the abbreviated Spanish version of the Motivated Strategies for Learning Questionnaire (MSLQ) developed by Pintrich and De Groot (1990). The MSLQ was created within a motivational and self-regulated socio-cognitive framework. This questionnaire includes 44 items, and the response choices are rated on a 7-point Likert-type scale. MSLQ is divided into two sections: $m o-$ tivation and self-regulated learning strategies (SRLS). The sample included 318 students total who belong the Psychology department of Universidad de la República (Uruguay). Results have shown that both motivation ( $\alpha=$ $.76)$ and self-regulated learning strategies $(\alpha=.75)$ sections have acceptable internal consistency to be used in research studies. Although the exploratory factor analysis confirms the original five-factor structure, we suggest a reorganization of the self-regulated learning strategies section.
\end{abstract}

Keywords: $M S L Q$, validation, motivation, self-regulated learning, Psychology

\footnotetext{
*Correspondencia a: Karina Curione. Dirección postal: Tristán Narvaja 1674, CP 11200, Montevideo, Uruguay. Teléfono: (+598) 24008555. kcurione@ psico.edu.uy

Cómo citar este artículo: Curione, K., Gründler, V., Píriz, L., \& Huertas, J. A. (2017). MSLQ-UY, validación con estudiantes universitarios uruguayos. Revista Evaluar, 17(02), 1-17. Recuperado de https://revistas.unc.edu.ar/index.php/revaluar

Nota del autor: Este trabajo forma parte de la tesis de doctorado de la primera autora. Doctorado en Psicología, Universidad Católica del Uruguay.
} 


\section{Introducción}

Uno de los principales antecedentes en el estudio de la motivación, la autorregulación y el rendimiento académico a nivel internacional son los diversos estudios realizados desde mediados de la década de 1980 por Paul Pintrich y colaboradores (Pintrich \& De Groot, 1990; Pintrich, Smith, García, \& McKeachie, 1991,1993) los que condujeron a la creación del Motivated Strategies for Learning Questionnaire (MSLQ). El desarrollo de dicho instrumento comenzó en 1986 y supuso 10 años de trabajo de Pintrich y su equipo; durante ese tiempo, el instrumento fue utilizado en numerosos estudios correlacionales que involucraron a miles de estudiantes en sus muestras.

El MSLQ fue creado a partir del marco socio-cognitivo de la motivación y la autorregulación, el cual asume que la motivación está relacionada con la habilidad de autorregular las actividades de aprendizaje. Así, se considera que un estudiante necesita estar motivado para utilizar eficazmente las estrategias de aprendizaje (Pintrich \& De Groot, 1990). El cuestionario se utiliza para evaluar la motivación y las estrategias de aprendizaje autorregulado que el estudiante emplea en un curso en particular.

Pintrich (2000) define el aprendizaje autorregulado como un proceso activo, constructivo, mediante el cual los aprendices eligen metas para su aprendizaje e intentan monitorear, regular y controlar su cognición, su motivación y sus conductas, guiados por las constricciones de sus propias metas y de los factores contextuales y ambientales. La investigación en el área ha permitido caracterizar al aprendiz autorregulado como alguien autónomo, reflexivo y eficiente, que posee las habilidades cognitivas y metacognitivas, así como las creencias motivacionales y las actitudes necesarias para comprender, monitorear y dirigir su propio aprendizaje (Wolters, 2003).
Las subescalas motivacionales del MSLQ se basan en tres constructos motivacionales: expectativa, valor y afecto. El componente expectativa hace referencia a las creencias de los estudiantes acerca de sus propias habilidades para desempeñarse en una tarea. El componente valor incluye las metas de los estudiantes y sus creencias acerca de la importancia e interés de la tarea. Por último, el componente afectivo remite a las reacciones emocionales de los estudiantes ante la tarea (Pintrich \& De Groot, 1990).

El MSLQ en su versión abreviada (Pintrich $\&$ De Groot, 1990), objeto de la presente validación, contiene 44 ítems que se responden con una escala de tipo Likert cuyo rango de respuestas abarca de 1 a 7 . El cuestionario se divide en dos partes, denominadas motivación y estrategias de aprendizaje autorregulado (EAA). Estas a su vez se dividen en subescalas. Motivación se divide en autoeficacia, valor intrínseco y ansiedad ante las pruebas; estrategias de aprendizaje autorregulado, por su parte, se divide en uso de estrategias cognitivas y autorregulación.

Duncan y McKeachie (2005), dos protagonistas directos del proceso de creación del MSLQ, llevaron a cabo una revisión que incluyó 56 artículos en los que se empleaba el MSLQ. Estos autores identificaron solo tres artículos en los que se utilizaba la versión abreviada (44 ítems), 22 en los que se empleaba la versión completa (81 ítems) y otros 15 trabajos en los que se usaba o bien el bloque de motivación (7) o bien el bloque de estrategias de aprendizaje autorregulado (8). Estos datos hacen evidente lo señalado por Hilpert, Stempien, Van der Hoeven-Kraft y Husman (2013), que en pocos estudios se han examinado todas las subescalas en una única administración del MSLQ y en menos trabajos se ha estudiado la versión abreviada completa, objeto de la presente validación.

Según ha evidenciado la revisión realizada por Mayer, Faber y Xu (2007), el MSLQ es el 
instrumento más utilizado para evaluar la motivación en contextos educativos. Así, el MSLQ se ha convertido en un instrumento estándar en la investigación en el campo de la motivación y la autorregulación (Schunk, 2005).

El cuestionario MSLQ ha sido traducido a diferentes idiomas y validado en múltiples contextos (Erturan-Ilker, Arslan, \& Demirhan, 2014; Jakešová \& Hrbáčková, 2013; RamirezDorantes, Canto y Rodríguez, Bueno-Alvarez, \& Echazarreta-Moreno, 2013; Lee, Yin, \& Zhang, 2010; Rao \& Sachs, 1999). Además, se ha utilizado como medida criterio en estudios de validez convergente de nuevos instrumentos (este es el caso de Magno, 2011) y de instrumentos ya existentes como el LASSI (Learning and Study Strategies Inventory) en el caso del estudio llevado adelante por Muis, Winne y Jamieson-Noel (2007).

También se ha estudiado la capacidad del MSLQ para predecir el rendimiento académico, siendo el trabajo más relevante al respecto el meta-análisis efectuado por Credé y Phillips (2011). Los autores encontraron que las subescalas de regulación del esfuerzo, autoeficacia y gestión del tiempo y el ambiente de estudio muestran una relación (entre débil y moderada) con el rendimiento. Por su parte, las estrategias más específicas relacionadas con la actividad de estudio (como repetición, elaboración, organización, aprendizaje entre pares, etc.) son las menos predictivas del rendimiento académico. Esto ha sido explicado de distintas maneras por Credé y Phillips (2011).

En primer lugar señalan que algunas estrategias de aprendizaje, como por ejemplo el aprendizaje entre pares, pueden guardar una relación curvilínea con el rendimiento académico que no llega a ser capturada por los coeficientes de correlación. Tanto los estudiantes de bajo como de alto rendimiento pueden no recurrir al aprendizaje entre pares por distintas razones: los primeros pue- den no estar motivados para hacerlo, en tanto que los segundos pueden considerar no necesitarlo. Otra explicación planteada por estos autores para entender la falta de relación entre las estrategias de aprendizaje específicas y el rendimiento académico tiene que ver con el modo en que se evalúa el rendimiento académico. Frecuentemente, los estudiantes no necesitan resolver pruebas empleando estrategias de aprendizaje que supongan la necesidad de un procesamiento profundo de la información (p. ej.: elaboración). Los autores plantean de modo atinado que las evaluaciones de múltiple opción pueden requerir memorización, pero no evalúan el pensamiento crítico, y los estudiantes que se comprometen con el pensamiento crítico no necesariamente obtienen mejores resultados en las pruebas de múltiple opción.

Pintrich et al. (1993), al cierre del artículo en el cual presentaban la versión completa del MSLQ (81 ítems), señalaron:

Estas significativas aunque moderadas relaciones con las notas de los cursos, son razonables, dados todos los otros factores que se relacionan con las notas de los cursos a nivel universitario y que no son medidos por el MSLQ (las notas individuales del curso en sí mismas no son medidas confiables del rendimiento o del aprendizaje) (p. 812).

Estas consideraciones muestran que, más allá de la complejidad de las relaciones entre los constructos del MSLQ, se sitúa la problemática de la evaluación del rendimiento en el nivel académico.

Al retomar el tema del volumen de producción asociado al MSLQ, se constata otro conjunto de estudios en los que se han analizado las propiedades psicométricas de este instrumento (Pintrich et al., 1993; Hilpert et al., 2013; Dunn, Lo, Mulvenon, \& Sutcliffe, 2012); de modo sistemático este instrumento ha mostrado ser una me- 
dida confiable de la motivación y las estrategias de aprendizaje autorregulado. Como lo señalaran Duncan y McKeachie (2005), el MSLQ se caracteriza por poseer una estructura conceptual sólida que brinda sustento a su construcción.

Sin embargo, también cabe mencionar que varios estudios han informado problemas vinculados a la estructura factorial latente y han sugerido importantes modificaciones. En particular Hilpert et al. (2013), tras reespecificar la estructura factorial del MSLQ, y luego de explorar varios modelos mediante análisis factorial confirmatorio, propusieron remover más de la mitad de las subescalas, de modo que el modelo final quedó compuesto por tres factores con dos subescalas cada uno: expectativa (autoeficacia y control del aprendizaje), valor (metas intrínsecas y valor de la tarea) y autorregulación (regulación metacognitiva y regulación del esfuerzo).

Dunn et al. (2012) se plantearon el objetivo de evaluar críticamente las dos subescalas de autorregulación, a saber: autorregulación metacognitiva y regulación del esfuerzo. Los resultados de dicho estudio mostraron que la estructura de dos subescalas de autorregulación no tendría suficiente soporte. Los autores plantearon, por lo tanto, dos subescalas modificadas: la escala general de estrategias de aprendizaje (EGEA) y la escala de estrategias de clarificación para el aprendizaje (ECA). Por su parte, Hilpert et al. (2013) señalaron que la combinación propuesta por Dunn et al. (2012) mostró un buen ajuste con sus datos, e indicaron que esta modificación podría resultar útil a otros investigadores interesados en evaluar la autorregulación. Ambos estudios se llevaron a cabo con la versión completa del MSLQ (81 ítems; Pintrich et al., 1991; Pintrich et al., 1993).

En la presentación de la versión abreviada que fue objeto de la presente revisión, Pintrich y De Groot (1990) no informaron los análisis factoriales realizados, se limitaron a mencionar que:
"El análisis factorial fue utilizado para guiar la construcción de la escala, y resultó en la exclusión de algunos ítems debido a la falta de correlación o de una estructura factorial estable" ( $p$. 34). Los autores señalaron haber comenzado con 56 ítems, y la escala abreviada quedó finalmente conformada por 44 ítems. Si bien presentaron los análisis de confiabilidad, de correlación entre subescalas y las correlaciones del MSLQ con rendimiento académico, no se dispone de los análisis factoriales exploratorios ni confirmatorios de la versión abreviada. Algunos autores han criticado esta falta de especificación del modelo al mismo tiempo que han señalado problemas con la estructura latente (Dunn et al., 2012).

Más allá de los análisis críticos del MSLQ, el instrumento ha sido utilizado en cientos de estudios y en muy diversos contextos, mostrando sistemáticamente su consistencia como medida de la motivación y las estrategias de aprendizaje autorregulado. También se ha señalado su capacidad de predecir el rendimiento académico (Hilpert et al., 2013), siendo esto último uno de los principales intereses de Pintrich y su equipo.

A 27 años de su creación, el MSLQ sigue suscitando un interés permanente, lo que muestra su vigencia, por este motivo, se consideró importante contar con una validación al español de la versión abreviada de este instrumento para el contexto uruguayo.

\section{Método \\ Participantes}

La muestra utilizada en esta investigación estuvo integrada por 318 estudiantes de primer año de la Licenciatura en Psicología (Universidad de la República), 253 mujeres (80\%) y 65 hombres $(20 \%)$. La media de edad de los sujetos fue de 23.38 años, con un desvío típico de 4.2 años. 
Si bien se trabajó con una muestra de conveniencia, se realizó un muestreo por turnos para alcanzar mayor representatividad. Para esto se aplicó el cuestionario en el salón de clases en las cuatro franjas horarias en las que se brindaba la unidad curricular obligatoria Psicología del Desarrollo (segundo semestre del plan de estudios de la Licenciatura en Psicología, 2013). La muestra final mantiene las proporciones de la distribución por sexo y la media de edad que caracterizan a los estudiantes que ingresan cada año a la Facultad de Psicología.

\section{Instrumentos}

El MSLQ en su versión abreviada contiene 44 ítems que se responden con una escala de tipo Likert de 1 a 7 . Dicho cuestionario se divide en dos bloques, denominados motivación y estrategias de aprendizaje autorregulado. A continuación se presentan las subescalas y los coeficientes alfa de Cronbach presentados por Pintrich y De Groot (1990). El bloque de motivación incluye las subescalas: autoeficacia $(\alpha=.89)$, valor intrínseco $(\alpha=.87)$ y ansiedad ante las pruebas $(\alpha$ $=.75)$. Por otro lado, el bloque de estrategias de aprendizaje autorregulado incluye las siguientes subescalas: uso de estrategias cognitivas $(\alpha=.83)$ y autorregulación $(\alpha=.74)$.

Se administró la versión uruguaya del MSLQ-UY a todos los sujetos participantes. Se mantuvo la estructura de la versión original en inglés integrada por los siguientes 44 ítems, distribuidos en dos bloques (Tablas 1 y 2).

Rendimiento Académico. Como medida de rendimiento académico se tomó la nota final obtenida por los participantes en el curso Psicología del Desarrollo. Dicha nota surgió del promedio de las notas obtenidas por los estudiantes en dos pruebas parciales.
Tabla 1

Distribución de ítems del Bloque Motivación según subescala.

\begin{tabular}{lcc}
\hline Bloque Motivación & $\mathbf{N}^{\mathbf{0}}$ de Ítems & Total \\
\hline Valor Intrínseco & $1,4,5,7,10,14,15,17,21$ & 9 \\
Autoeficacia & $2,6,8,9,11,13,16,18,19$ & 9 \\
$\begin{array}{lcc}\text { Ansiedad } \\
\text { ante las Pruebas }\end{array}$ & $3,12,20,22$ & 4 \\
\hline Total & & 22
\end{tabular}

Tabla 2

Distribución de ítems del Bloque Estrategias de Aprendizaje Autorregulado según subescala.

\begin{tabular}{lcc}
\hline $\begin{array}{c}\text { Bloque Estrategias } \\
\text { de Aprendizaje } \\
\text { Autorregulado }\end{array}$ & $\mathbf{N}^{\mathbf{0}}$ de Ítem & Total \\
\hline Uso de Estrategias & $23,24,26,28,29,30,31$, & 13 \\
Cognitivas & $34,36,39,41,42,44$ & \\
Autorregulación & $25,27,32,33,35$, & 9 \\
\hline Total & $37,38,40,43$ & 22
\end{tabular}

\section{Procedimiento}

Traducción al español de la versión abreviada del MSLQ. El proceso de traducción y adaptación transcultural de la versión abreviada del MSLQ fue realizado según lo propuesto por la Comisión Internacional de Tests (International Test Commission [ITC], 2005), por Van Widenfelt, Treffers, de Beurs, Siebelink y Koudijs (2005) y por Muñiz, Elosua y Hambleton (2013). Participaron en total seis traductores competentes, quienes trabajaron por separado. Dos traductoras bilingües, ambas con formación en motivación en contextos educativos, tradujeron el instrumento del idioma inglés al español. Una vez obtenidas ambas versiones otras dos traductoras bilingües, ambas con formación en psicología y educación y hablantes nativas de inglés, realizaron la traducción inversa de las mismas. En este proceso de retrotraducción se identificaron ítems problemáticos que sufrieron 
modificaciones en el proceso de vuelta al idioma original. Por lo tanto, se solicitó a otros dos traductores profesionales, quienes no conocían este instrumento de medición, la traducción directa del inglés al español de los ítems problemáticos. La versión resultante del instrumento fue administrada a 40 estudiantes de Psicología, a quienes se les solicitó su opinión sobre el instrumento en general, así como respecto a la redacción de los ítems. Este paso fue fundamental para la realización de pequeños ajustes. A partir de este proceso se obtuvo la versión en español del MSLQ.

Finalmente, el instrumento en versión papel fue administrado a los estudiantes que participaron del estudio. La aplicación se realizó en el contexto natural del salón de clases de Psicología del Desarrollo, unidad curricular obligatoria del primer año de la Licenciatura en Psicología. Los estudiantes completaron el MSLQ-UY en presencia de la docente del curso antes del comienzo de la clase; fueron informados de los objetivos del estudio e invitados a participar de manera libre y voluntaria, y manifestaron su aceptación mediante la firma de un consentimiento libre e informado. Este estudio contó con el aval del Comité de Ética en Investigación de la Facultad de Psicología. La aplicación del cuestionario insumió entre 20 y 25 minutos.

Análisis Psicométricos. Los datos fueron ingresados y procesados utilizando el paquete estadístico SPSS versión 15.0 (IBM Corporation, 2006) y en primer lugar se analizaron datos descriptivos de la población (sexo y edad). Para llevar adelante el estudio psicométrico de la escala se tomaron como referencia las indicaciones de Carretero-Dios y Pérez (2005). Se realizó un análisis de los ítems para verificar si se ajustaban adecuadamente a la escala.

Se verificó el promedio de los ítems y su desviación estándar con respecto a la media de la escala. De acuerdo con las recomendaciones de Carretero-Dios y Pérez (2005) se consideraron adecuados aquellos ítems con una desviación típica superior a 1 y una media situada alrededor del punto medio de la escala (simetría próxima a 0 ).

Para calcular el nivel de discriminación de cada ítem se recurrió al coeficiente de correlación corregido entre la puntuación del ítem y la total obtenida en la dimensión a la que este pertenece. Se consideraron adecuados valores entre 0.250.30 (Nunnally \& Bernstein, 1995).

Se verificaron los coeficientes de asimetría y se consideraron adecuados aquellos valores entre -1 y + 1 (Ferrando \& Anguiano-Carrasco, 2010).

Para analizar la consistencia interna de la escala se obtuvieron los coeficientes alfa de Cronbach para los dos bloques y sus correspondientes dimensiones. Para estudiar la dimensionalidad de la escala se llevó adelante un análisis factorial exploratorio (AFE) mediante la técnica de extracción por componentes principales y rotación varimax. Para confirmar el número de factores a extraer se consideró el gráfico de sedimentación (Cattell, 1966).

\section{Resultados \\ Estructura factorial}

Se calculó la medida de adecuación muestral KMO (Kaiser-Meyer-Olkin) para comprobar si los valores muestrales permitían o no la aplicación del análisis factorial. El estadístico KMO varía entre 0 y 1 ; valores menores que .5 indican que los datos que se están analizando no son aptos para efectuar un análisis factorial. La medida KMO fue .806 , por lo cual se procedió a realizar el análisis.

Por otro lado, la prueba de esfericidad de Bartlett contrasta la hipótesis nula de que la matriz de correlaciones es una matriz de identidad. 
Tabla 3

Análisis factorial exploratorio.

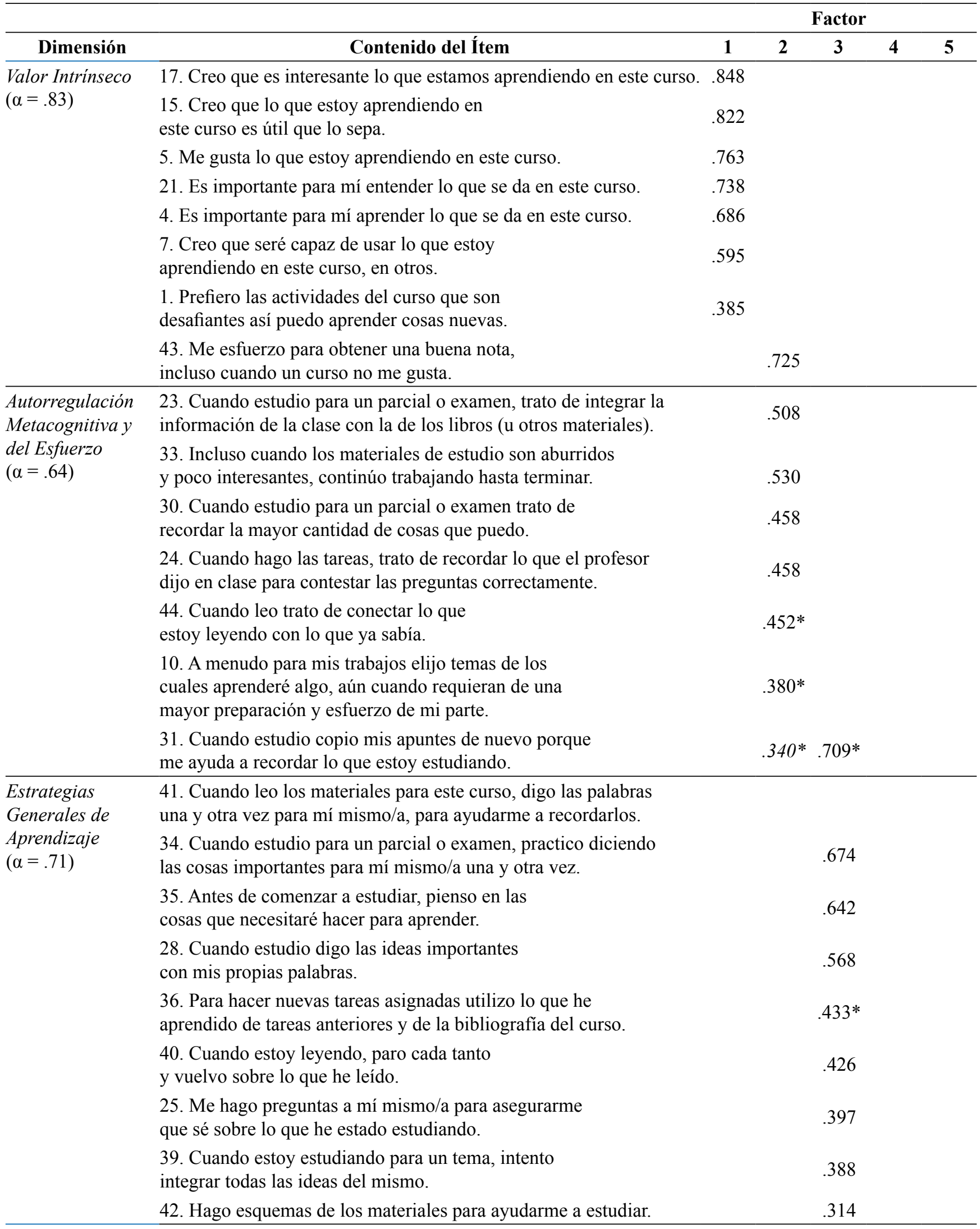




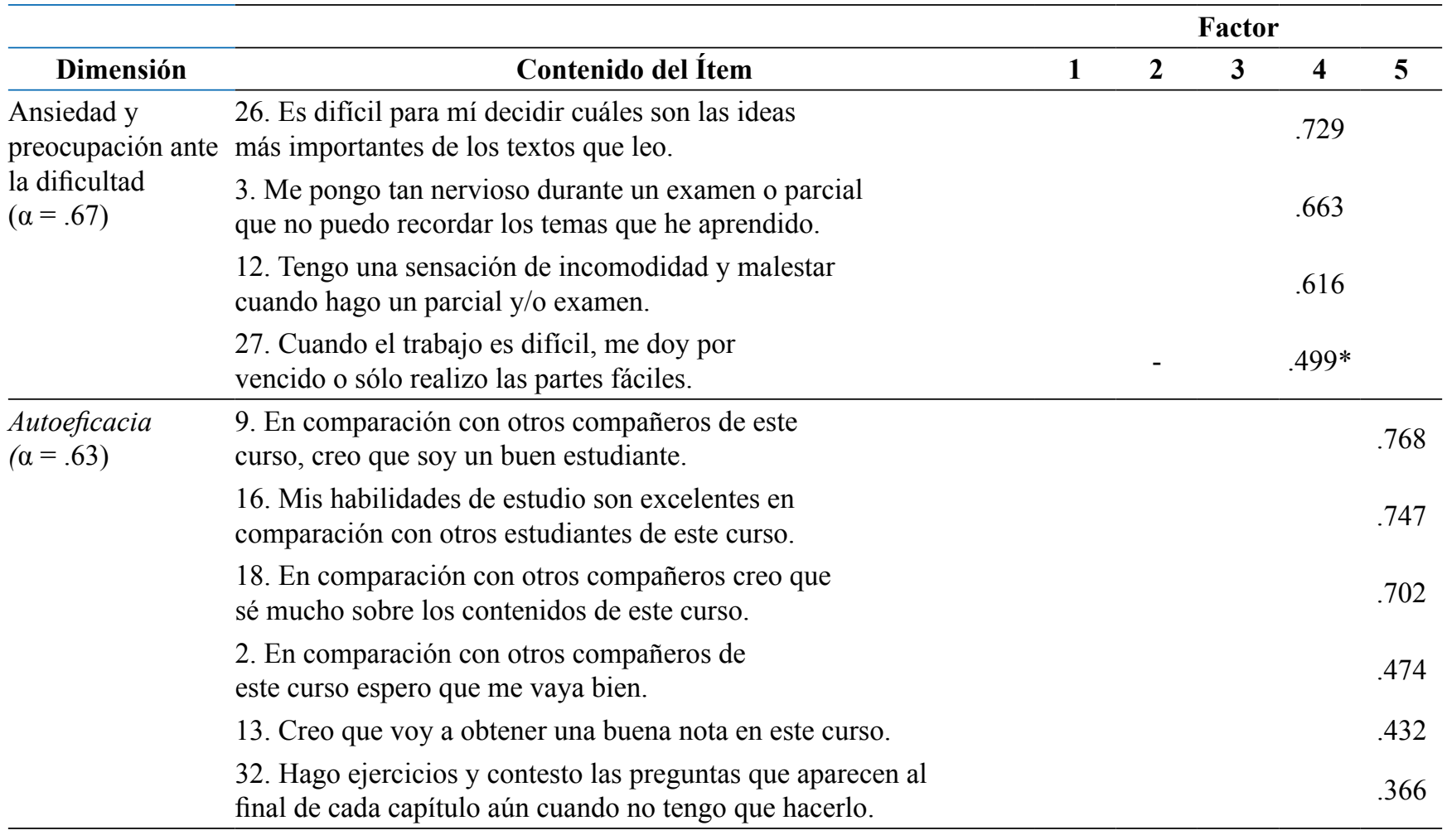

Nota. Método de extracción: análisis de componentes principales; método de rotación: normalización varimax con Kaiser. La rotación ha convergido en 7 iteraciones. * Ítems que presentan solapamiento en dos dimensiones. Se los ubicó en la dimensión con mayor carga factorial y sentido teórico.

Si el nivel crítico es mayor a .05 no es posible rechazar la hipótesis nula de esfericidad; por lo tanto, no es posible asegurar que el modelo factorial sea adecuado para explicar los datos. Se obtuvo un valor de $p<.001$ en el test de esfericidad de Bartlett. La hipótesis nula fue rechazada, por lo que fue procedente aplicar el análisis factorial.

Se efectuó un análisis factorial exploratorio (AFE), con el método de componentes principales y rotación varimax. El gráfico de sedimentación (Cattell, 1966) permitió visualizar cinco factores principales. El punto de corte en la tendencia descendente se tomó como criterio para la determinación del número óptimo de factores que deben estar presentes en la solución factorial (Pardo \& Ruiz, 2002).

Los resultados de este estudio brindan evidencia a favor de la estructura de cinco dimensiones de la escala original. La totalidad de los ítems presentaron cargas por encima de .30 , y se observaron valores aceptables en las comunalidades de todos los ítems $\left(h^{2}>.20\right)$, siendo la menor comunalidad .21 (ítem 38) y la mayor comunalidad 69 (ítem 15). Al llevar adelante el primer AFE, la matriz de componentes rotados evidenció varios ítems con cargas compartidas: $6,8,11,14$, 19, 27, 32, 37. Por su parte, el ítem 37 presentó el coeficiente de correlación inter-ítem más bajo de su componente $(r=-.036)$ y el ítem 22 presentó el más bajo coeficiente de correlación inter-ítem del bloque motivacional $(r=.007)$. Se eliminaron los ítems solapados y el ítem 22 por su baja capacidad de discriminación. Tras la eliminación de estos ítems se realizó un nuevo AFE.

Se encontraron para la estructura factorial del MSLQ-UY cinco factores que explican el $44.28 \%$ de la varianza:

Factor 1 (valor intrínseco): ítems 1, 4, 5, 7, 15, 17, 21 (siete de los nueve originales), este factor resultó el más estable de la estructura MSLQ. 
Factor 2 (autorregulación -metacognitiva y del esfuerzo-): ítems 10, 23, 24, 29, 30, 31, 33, 43, 44.

Factor 3 (estrategias generales de aprendizaje): ítems 25, 28, 34, 35, 36, 39, 40, 41, 42.

Factor 4 (ansiedad y preocupación ante la dificultad): ítem 3, 12, 20, 26, 27.

Factor 5 (autoeficacia): ítems 2, 9, 13, 16, 18,32 .

\section{Consistencia interna}

Se procedió a calcular la confiabilidad del MSLQ-UY por componente y por subescala a partir de la estructura factorial resultante. Los resultados se presentan a continuación.

Tabla 4

Análisis de confiabilidad por subescala según componentes del MSLQ-UY.

\begin{tabular}{lc}
\hline & Coeficiente $\alpha$ \\
\hline Componente Motivacional & $\mathbf{. 7 6}$ \\
\hline Valor Intrínseco & .83 \\
Autoeficacia & .63 \\
Ansiedad ante las Pruebas & .67 \\
\hline Componente Aprendizaje & .75 \\
Autorregulado & .71 \\
\hline Estrategias Generales de Aprendizaje & .64 \\
\hline Autorregulación & \\
\hline
\end{tabular}

Los índices de confiabilidad de los componentes fueron aceptables para su uso con fines de investigación $(\alpha=.76$ y $\alpha=.75)$.

\section{Correlaciones entre factores}

Se estudiaron las relaciones entre los componentes motivacionales y de aprendizaje autorregulado del MSLQ-UY, y los resultados pueden observarse en la Tabla 5. Altos niveles de autoe- ficacia y valor intrínseco se relacionaron con altos niveles de uso de estrategias cognitivas y de autorregulación. El uso de estrategias cognitivas se relacionó con la autorregulación, y esta fue la correlación más elevada entre dimensiones del MSLQ-UY.

\section{Tabla 5}

Correlaciones entre dimensiones motivacionales y de aprendizaje autorregulado.

\begin{tabular}{lcccc}
\hline \multicolumn{1}{c}{ Dimensión } & $\mathbf{1}$ & $\mathbf{2}$ & $\mathbf{3}$ & $\mathbf{4}$ \\
\hline 1. Valor Intrínseco & - & & & \\
$\begin{array}{l}\text { 2. Autoeficacia } \\
\text { 3. Ansiedad ante }\end{array}$ & $.443^{* *}$ & - & & \\
$\begin{array}{l}\text { las Pruebas } \\
\text { 4. Uso de }\end{array}$ & .036 & -.038 & - & \\
$\begin{array}{l}\text { Estrategias } \\
\text { 5. Autorregulación }\end{array}$ & $.322^{* *}$ & $.375^{* *}$ & -.057 & $.545^{* *}$ \\
\hline
\end{tabular}

Nota. $* p<.05$ (bilateral), ${ }^{* *} p<.01$ (bilateral).

\section{Validez predictiva}

A los efectos de estudiar la validez predictiva del MSLQ-UY se tomó como criterio externo el rendimiento académico de los estudiantes (operativizado como la nota final promedio obtenida en el curso donde el instrumento fue aplicado). Se encontró una correlación positiva y significativa entre la subescala de autorregulación y la nota obtenida por los estudiantes $(r=.154 ; p<.01)$. El resultado es similar al obtenido mediante un modelo de análisis de regresión múltiple. La única variable de MSLQ-UY que incidió significativamente en el rendimiento, pero de manera muy moderada, fue la subescala de autorregulación $\left(\mathrm{R}^{2}\right.$ $=.037, \beta=.17 ; p<.01)$. Aquellos estudiantes que informaron mayores niveles de autorregulación del aprendizaje mostraron en este estudio una tendencia a obtener un mejor rendimiento. 
Validez convergente

Se estudió la validez convergente del MSLQ-UY con respecto a la versión española (Núñez, Martín-Albo, \& Navarro, 2005) de la Échelle de Motivation en Éducation (EME; Vallerand, Blais, Brière, \& Pelletier, 1989). La EME-E consiste en 28 ítems distribuidos en 7 subescalas de cuatro ítems cada una, que evalúan los tres tipos de motivación intrínseca (relativa al conocimiento, al logro y a las experiencias estimulantes), los tres tipos de motivación extrínseca (externa, introyectada e identificada) y la amotivación (Ryan \& Deci, 2000).

Se aplicó la EME-E a una submuestra de 119 participantes que habían completado el MSLQUY, 94 mujeres (79\%) y 25 varones $(21 \%)$. La media de edad de este grupo fue de 22.6, con una desviación típica de 6.8 años.

Se calcularon las correlaciones entre las subescalas del MSLQ y las subescalas que integran la EME-E. También se efectuaron análisis de regresión múltiple tomando como variable dependiente cada una de las escalas de la EME-E. Los resultados se presentan a continuación.

\section{Subescala de Motivación Intrínseca al Conoci-} miento (MIC) de EME-E. El análisis de regresión muestra que esta subescala recibió una incidencia significativa de grado moderado-bajo $\left(\mathrm{R}^{2}=.21\right)$ de la subescala de autorregulación de MSLQ-UY $(\beta=.22, p<.001)$, la que a su vez correlacionó con MIC $(r=.40, p \leq .01)$.

Subescala de Motivación Intrínseca al Logro (MIL) de EME-E. El análisis de regresión mostró una capacidad predictiva moderada-baja $\left(\mathrm{R}^{2}=\right.$ .18) de las subescalas de autorregulación $(\beta=.26$; $p<.023) \mathrm{y}$ ansiedad ante las pruebas $(\beta=.20 ; p<$ .023) de MSLQ-UY sobre esta subescala. Por su parte, MIL correlacionó con autorregulación ( $r=$ $.33, p \leq .01)$ y con ansiedad ante las pruebas $(r=$
$.20, p \leq .05)$.

Subescala de Motivación Intrínseca a las Experiencias Estimulantes (MIEE). Volvió a aparecer un modelo de regresión con capacidad predictiva moderada-baja $\left(\mathrm{R}^{2}=.16\right)$ en el que sólo había una variable predictora, la subescala de autorregulación del MSLQ-UY $(\beta=.30 ; p<.01)$. Los resultados mostraron una correlación de autorregulación con MIEE $(r=.38, p \leq .01)$.

Subescala de Regulación Externa de EME-E. Se verificó un modelo de regresión que explicaba una parte moderada-baja de la varianza $\left(\mathrm{R}^{2}=.15\right)$ con dos variables predictoras: autorregulación $(\beta$ $=-.41 ; p<.001)$ y autoeficacia $(\beta=.24 ; p<.021)$. Subescala de Regulación Introyectada de $\boldsymbol{E M E}-\boldsymbol{E}$. Se obtuvo un modelo con capacidad predictiva moderada-baja $\left(\mathrm{R}^{2}=.14\right)$ con una variable predictora: ansiedad ante las pruebas $(\beta=.32 ; p<$ $.001)$. Se encontró también correlación entre ambas subescalas $(r=.34, p \leq .01)$.

Valor intrínseco del MSLQ-UY. Correlacionó con las tres subescalas de motivación intrínseca (MI) de la EME-E: motivación intrínseca al conocimiento $(r=.34, p \leq .01)$, al logro $(r=.20, p$ $\leq .01)$ y a las experiencias estimulantes $(r=.25$, $p \leq .01)$.

Uso de estrategias cognitivas del MSLQ-UY. Correlacionó con los tres tipos de motivación intrínseca: al logro $(r=.31, p \leq .01)$; al conocimiento $(r=.27, p \leq .01)$ y a las experiencias estimulantes $(r=.26, p \leq .01)$.

Autorregulación del MSLQ-UY. También correlacionó con los tres tipos de motivación intrínseca: al logro $(r=.33, p \leq .01)$; al conocimiento $(r$ $=.40, p \leq .01) \mathrm{y}$ a las experiencias estimulantes $(r$ $=.38, p \leq .01)$.

Regulación Externa. Es la más extrínseca de las motivaciones extrínsecas en el continuo de autodeterminación (Ryan \& Deci, 2000), y no co- 
rrelacionó con ninguna de las dimensiones del MSLQ-UY.

Amotivación de EME-E. Correlacionó de modo negativo con uso de estrategias cognitivas del MSLQ-UY ( $r=-.21, p \leq .05)$ y también correlacionó negativamente con autorregulación $(r=$ $-.24, p \leq .05)$.

Los resultados muestran la validez convergente entre dos instrumentos ampliamente utilizados para evaluar la motivación en contextos educativos.

\section{Discusión}

El objetivo de este trabajo fue presentar el proceso de traducción al español, adaptación y validación del MSLQ para el contexto universitario uruguayo. En este trabajo se presenta la primera etapa del trabajo llevado adelante, el cual se ha caracterizado por un riguroso proceso de traducción y adaptación del instrumento desde su versión original. Con respecto a la versión uruguaya del MSLQ (MSLQ-UY), tanto para el bloque motivación $(\alpha=.76)$ como el bloque estrategias de aprendizaje autorregulado $(\alpha=.75)$ se obtuvieron índices de consistencia interna que hacen recomendable su uso con fines de investigación. La menor confiabilidad del bloque motivación con respecto al instrumento original podría deberse a la disminución de la consistencia interna de la subescala de autoeficacia $(\alpha=.63)$ con respecto a la original $(\alpha=.89)$ informada por Pintrich y De Groot (1990).

La estructura factorial de cinco dimensiones propuesta por Pintrich y De Groot (1990) fue confirmada en este estudio; sin embargo fue necesario eliminar un total de 7 ítems y la versión final quedó conformada por 37 ítems. Las dos subescalas del bloque estrategias de aprendizaje autorregulado fueron las que presentaron más dificultades con respecto a su estructura factorial, ya que se encontraron varios ítems con cargas repartidas entre ambos componentes. La subescala de autorregulación ( $\alpha=.64)$ fue categorizada en este estudio como autorregulación del esfuerzo y autorregulación metacognitiva, dado que contiene ítems que evalúan distintas facetas del aprendizaje autorregulado, y en la versión completa del MSLQ (Pintrich et al., 1993) integra distintas dimensiones que evalúan la regulación del esfuerzo por un lado y la autorregulación metacognitiva por otro. La necesidad de reorganización de las subescalas del bloque EAA ha sido señalada en varios estudios previos (Rao \& Sachs, 1999; Lee et al., 2010; Dunn et al., 2012; Hilpert et al., 2013).

Se estudió la relación entre los componentes motivacionales y de aprendizaje autorregulado; las relaciones encontradas van en la misma dirección que las reportadas por Pintrich y De Groot (1990). Los estudiantes que se consideran más capaces (niveles elevados de autoeficacia) presentan mayores niveles de valor intrínseco y es más probable que utilicen estrategias cognitivas y de autorregulación ante la actividad de estudio. Sin embargo, en nuestro estudio no se encontraron las relaciones negativas entre ansiedad ante las pruebas y autoeficacia halladas por Pintrich y De Groot (1990). Esta discrepancia fue previamente informada por Rao y Sachs (1999) en uno de los pocos estudios que propuso validar la versión abreviada del MSLQ, en ese caso para el contexto chino. Estos autores señalan diferencias culturales entre los estudiantes asiáticos y los norteamericanos: en estudiantes chinos, la ansiedad ante las pruebas podría no afectar las creencias sobre sí mismos como aprendices del mismo modo en que afecta a los estudiantes norteamericanos.

Por su parte, Pintrich y De Groot (1990) no informaron relaciones entre ansiedad ante las pruebas y uso de estrategias cognitivas, mientras 
que en nuestro estudio ambas dimensiones mostraron estar positivamente relacionadas. Esta discrepancia podría deberse a diferencias culturales entre la muestra uruguaya y la muestra norteamericana; estudiantes ansiosos ante las evaluaciones podrían acudir en mayor medida al uso de estrategias cognitivas para la preparación de las evaluaciones. La relación positiva entre ansiedad ante las evaluaciones y uso de estrategias cognitivas también fue informada en el estudio con población china ya mencionado. Rao y Sachs (1999) plantearon en dicho trabajo que la ansiedad ante las evaluaciones podría tener cierto componente facilitador que lleve al uso eficiente de estrategias cognitivas.

Asimismo, la dimensión de uso de estrategias cognitivas contiene ítems que apuntan al uso de la repetición como estrategia de memorización (p. ej., Ítem 41, Cuando leo los materiales de este curso, digo las palabras para mí mismo una y otra vez para ayudarme a recordarlos). Estudios previos con el MSLQ han mostrado diferencias culturales en el empleo de la memorización a nivel universitario. Lee et al. (2010) plantearon que para los estudiantes chinos, la memorización y la comprensión se encuentran culturalmente relacionadas, en tanto que la cultura occidental por el contrario, tiende a disociar memorización de comprensión. Purdie y Hattie (1996) compararon el uso de estrategias de aprendizaje autorregulado en estudiantes japoneses y australianos, y encontraron que los estudiantes japoneses tendían a utilizar la memorización en mayor grado que los estudiantes australianos. Sin embargo, los estudiantes japoneses consideraban la memorización como un camino hacia la comprensión. Los educadores occidentales asumen que la comprensión es una estrategia superior a la repetición, y tienden a disociar ambos procesos; sin embargo las investigaciones con estudiantes de Asia Oriental han mostrado que la memorización para esos aprendices difiere cualitativamente de la memorización mecánica, y los estudiantes son alentados a memorizar para alcanzar un mayor comprensión (Rao \& Sachs, 1999).

Se estudió la validez predictiva del MSLQUY tomando como criterio externo el rendimiento académico de los estudiantes en el curso donde el instrumento fue aplicado. Aquellos estudiantes que informaron mayores niveles de autorregulación del aprendizaje tendieron a presentar un mejor rendimiento académico. Estos resultados son consistentes con los reportados por Pintrich y De Groot (1990). La incidencia de esta relación fue moderada-baja, similar a las relaciones de este tipo informadas en estudios previos con MSLQ (Pintrich \& De Groot, 1990; Pintrich et al., 1993; Rotgans \& Schmidt, 2010; Credé \& Phillips, 2011).

Para entender las débiles o moderadas relaciones entre las dimensiones del MSLQ y el rendimiento académico es importante recordar, en primer lugar, lo planteado por Pintrich et al. (1993), quienes puntualizaron que dichos resultados son razonables a la luz de todas las variables que inciden en el rendimiento y que no son evaluadas por dicho instrumento. Al mismo tiempo, los creadores del MSLQ alertaron acerca de que las calificaciones obtenidas en los cursos pueden no ser, por sí mismas, medidas confiables del rendimiento o el aprendizaje.

Como se señaló anteriormente, Credé y Phillips (2011) plantearon como hipótesis que las estrategias de aprendizaje más específicas (como el aprendizaje entre pares) podrían tener una relación curvilínea con el rendimiento que los coeficientes de correlación no logran capturar. Los autores también subrayaron el hecho de que las evaluaciones con formato de opción múltiple -que se aplican sobre todo al inicio de las carreras universitarias- evalúan de modo muy superficial el aprendizaje de los contenidos y no requerirían 
del uso de estrategias de procesamiento profundo de la información.

Al estudiar la validez convergente del MSLQ-UY con la Escala de Motivación Educativa se encontró que las relaciones entre las subescalas de ambos instrumentos van en la dirección teórica esperada. Valor intrínseco del MSLQ-UY correlacionó con las tres subescalas de motivación intrínseca de la EME-E: motivación intrínseca al conocimiento, al logro y a las experiencias estimulantes, lo cual tiene sentido dada la cercanía conceptual de dichos constructos. Pintrich y De Groot (1990) mencionan que la dimensión valor involucra las metas de los estudiantes al abordar una tarea y las creencias acerca de la importancia y el interés de dicha tarea; reconocen que esta dimensión puede ser conceptualizada de diferentes modos, como metas de aprendizaje versus metas de rendimiento, orientación intrínseca versus extrínseca, valor de la tarea o interés intrínseco. Los ítems del MSLQ fueron adaptados, según los autores, desde diversos instrumentos, entre los que mencionan la Escala de Orientación Intrínseca vs. Extrínseca en el Salón de Clases de Harter (1981).

Por su parte, uso de estrategias cognitivas del MSLQ-UY correlacionó con los tres tipos de motivación intrínseca (logro, conocimiento y experiencias estimulantes). La subescala autorregulación del MSLQ-UY también correlacionó con los tres tipos de motivación intrínseca. Estos resultados se relacionan con los obtenidos por Pintrich y De Groot (1990), y Pintrich et al. (1993), los cuales indican que altos niveles de valor intrínseco se relacionan con el uso de estrategias cognitivas y la autorregulación. Otros estudios han señalado que los estudiantes motivados intrínsecamente tienden a utilizar estrategias de procesamiento más profundo de la información (Karagüven, 2012). Maralani (2016), en un estudio en el cual se utilizaron ambos instrumentos, halló un rol mediador de la motivación intrínseca en la autorregulación metacognitiva; según este estudio, los ambientes educativos promotores de creatividad incrementarían la motivación intrínseca y, en consecuencia, el uso de estrategias de autorregulación metacognitivas por parte de los estudiantes.

En el presente estudio, ansiedad ante las pruebas del MSLQ-UY correlacionó con la subescala de motivación extrínseca regulación introyectada. En este tipo de orientación motivacional el estudiante actúa por la presión proveniente del exterior, y realiza las actividades educativas para evitar sentirse culpable y como modo de preservar su autoestima (Ryan \& Deci, 2000). A nivel teórico tiene sentido que la ansiedad ante las pruebas prediga este estilo de regulación, que es en parte externo.

Regulación externa (la más extrínseca de las motivaciones extrínsecas en el continuo de autodeterminación) no correlacionó con ninguna de las dimensiones del MSLQ-UY, lo cual tiene sentido si se considera que este instrumento en su versión abreviada no contiene la dimensión orientación extrínseca, limitación que había sido señalada por Lee et al. (2010).

Por último, la subescala amotivación de EME-E correlacionó de modo negativo con uso de estrategias cognitivas de MSLQ-UY y también correlacionó negativamente con autorregulación. La amotivación es definida como el estado de falta de intención para actuar (Ryan \& Deci, 2000), de modo que estas correlaciones van en la dirección esperada. Como Pintrich y De Groot (1990) señalaron, los estudiantes no sólo tienen que disponer de ciertas estrategias de aprendizaje, sino que también necesitan estar motivados para emplearlas.

En síntesis, se ha presentado evidencia de la validez convergente entre dos instrumentos ampliamente utilizados para evaluar la motivación en contextos educativos. Especialmente se 
encontró que la subescala de autorregulación del MSLQ-UY presenta claras relaciones con los factores de motivación intrínseca del EME-E, y ansiedad ante las pruebas con los factores más extrínsecos de dicha escala.

\section{Conclusiones}

Se verificaron índices de consistencia interna para la versión uruguaya del MSLQ que hacen recomendable su uso con fines de investigación, si bien aún no se ha comprobado la conveniencia de su uso con fines diagnósticos. Se presentaron evidencias de su validez estructural y de su validez convergente con una medida motivacional ampliamente utilizada (EME-E).

Es posible afirmar que el MSLQ-UY es un instrumento adecuado para evaluar la motivación académica y las estrategias de aprendizaje autorregulado que emplean los estudiantes universitarios. No obstante, para permitir su uso más allá de los contextos de investigación y aportar a la evaluación y la intervención psicoeducativa se requiere continuar trabajando en la mejora de este instrumento.

En este trabajo se identificaron problemas con la subescala de autoeficacia, la cual requiere mejoras en la adaptación cultural de aquellos ítems que apuntan a evaluar este constructo en función de un criterio competitivo que es habitual en el sistema educativo norteamericano, pero que resulta poco ajustado al perfil de los estudiantes de una universidad pública latinoamericana de libre acceso. Por ejemplo, el ítem 9 propone: En comparación con otros compañeros de este curso creo que soy un buen estudiante. Se recomienda, para futuras investigaciones en nuestro contexto, la reformulación de estos ítems orientada a la evaluación del componente centrada en el propio desempeño.
Entre las limitaciones de este estudio se encuentra el haber trabajado con estudiantes de una única carrera; es necesario continuar analizando las propiedades psicométricas del MSLQ-UY, ampliando la muestra a estudiantes de otras carreras universitarias e incluyendo asignaturas que utilicen distintos formatos de evaluación. Asimismo, se deberán efectuar otros análisis, en particular un análisis factorial confirmatorio para poner a prueba la estructura teórica del instrumento.

En lo que refiere a la medición del aprendizaje autorregulado, el MSLQ se ha convertido en una medida estándar; sin embargo enfrenta, como todo cuestionario de autoinforme, el problema de ser administrado antes o después de una tarea y no durante la realización de la misma. Esto conlleva dificultades asociadas a la falta de conciencia de los procesos autorregulatorios, a la reconstrucción a partir de la memoria para dar una respuesta sobre una tarea pasada, problemas de comprensión lectora, etc. (Veenman, 2011). Estudios futuros deberían poner en relación el MSLQ con otros tipos de medidas del aprendizaje autorregulado (observacionales, entrevistas, protocolos de pensamiento en voz alta, etc.).

Por último, es necesario mejorar la adecuación del MSLQ al modo en que los estudiantes abordan la actividad de estudio en un contexto donde la tecnología ocupa un lugar cada vez más relevante en la vida cotidiana, ya que ninguna subescala de este instrumento permite evaluar el aprendizaje mediado por nuevas tecnologías (Curione \& Huertas, 2016). Los cambios sociales ocurridos desde la aparición del MSLQ justifican la necesidad de este tipo de revisión (Dunn et al., 2012).

Más allá de los ajustes que es necesario realizar, el MSLQ se presenta como una medida confiable que ha mostrado una vez más su posibilidad de adaptación a distintos contextos educativos. 


\section{Referencias}

Carretero-Dios, H., \& Pérez, C. (2005). Normas para el desarrollo y revisión de estudios instrumentales. International Journal of Clinical and Health Psychology, 5(3), 521-551. Recuperado de http:// www.aepc.es/ijchp

Credé, M., \& Phillips, L. A. (2011). A meta-analytic review of the Motivated Strategies for Learning Questionnaire. Learning and Individual Differences, 21(4), 337-346. doi: 10.1016/j.lindif.2011.03.002

Cattell, R. B. (1966). The scree test of the number of significant factors. Multivariate Behavioral Research, 1, 140-161. Recuperado de http://www.tandfonline. com/doi/abs/10.1207/s15327906mbr0102_10

Curione, K., \& Huertas, J. A. (2016). Revisión del MSLQ: Veinticinco años de evaluación motivacional. Revista de Psicología, 12(24), 55-67. Recuperado de www. uca.edu.ar/revistadepsicologia

Duncan, T. G., \& McKeachie, W. J. (2005). The making of the Motivated Strategies for Learning Questionnaire. Educational Psychologist, 40(2), 117-128. doi: 10.1207/s15326985ep4002_6

Dunn, K. E., Lo, W.-J., Mulvenon, S. W., \& Sutcliffe, R. (2012). Revisiting the Motivated Strategies for Learning Questionnaire: A theoretical and statistical reevaluation of the metacognitive self-regulation and effort regulation subscales. Educational \& Psychological Measurement, 72(2), 312-331. doi: 10.1177/0013164411413461

Erturan-Ilker, G., Arslan, Y., \& Demirhan, G. (2014). A validity and reliability study of the Motivated Strategies for Learning Questionnaire. Educational Sciences: Theory Practice, 14(3), 829-833. doi: 10.12738/ estp.2014.3.1871

Ferrando, P. J., \& Anguiano-Carrasco, C. (2010). El análisis factorial como técnica de investigación en psicología. Papeles del Psicólogo, 31(1), 18-33. Recuperado de http://www.papelesdelpsicologo.es/

Harter, S. (1981). A new self-report scale of intrinsic versus extrinsic orientation in the classroom:
Motivational and informational components. Developmental Psychology, 17(3), 300-312. doi: 10.1037/0012-1649.17.3.300

Hilpert, J. C., Stempien, J., Van der Hoeven-Kraft, K. J., \& Husman, J. (2013). Evidence for the latent factor structure of the MSLQ: A new conceptualization of an established questionnaire. SAGE Open, 3(4), 1-10. doi: $10.1177 / 2158244013510305$

IBM. (2006). SPSS (Versión 15) [software]. Armonk, NY: IBM Corp.

International Test Commission (2005). International Guidelines on Test Adaptation. Recuperado de http:// www.intestcom.org

Jakešová, J., \& Hrbáčková, K. (2014). The Czech adaptation of Motivated Strategies for Learning Questionnaire (MSLQ). Asian Social Science, 10(12), 72-78. doi: 10.5539/ass.v10n12p72

Karagüven, M. H. Ü. (2012). The adaptation of Academic Motivation Scale to Turkish. Educational Sciences: Theory \& Practice, 12(4), 2611-2618. Recuperado de http://files.eric.ed.gov/fulltext/EJ1002866.pdf

Lee, J. C.-K., Yin, H. B., \& Zhang, Z. H. (2010). Adaptation and analysis of Motivated Strategies for Learning Questionnaire in the Chinese setting. International Journal of Testing, 10(2), 149-165. doi: 10.1080/15305050903534670

Magno, C. (2011). Validating the Academic Selfregulated Learning Scale with the Motivated Strategies for Learning Questionnaire (MSLQ) and Learning and Study Strategies Inventory (LASSI). The International Journal of Educational and Psychological Assessment, 7(2), 56-73. Recuperado de https://sites.google.com/site/tijepa2012/home

Maralani, F. M. (2016). The mediation role of intrinsic and extrinsic motivation in the relationship between creative educational environment and metacognitive self-regulation. Journal of Education and Learning, 5(3), 272-277. doi: 10.5539/jel.v5n3p272

Mayer, J. D., Faber, M. A., \& Xu, X. Y. (2007). Seventyfive years of motivation measures (1930-2005): A descriptive analysis. Motivation and Emotion, 31(2), 
83-103. doi: 10.1007/s11031-007-9060-2

Muis, K. R., Winne, P. H., \& Jamieson-Noel, D. (2007). Using a multitrait-multimethod analysis to examine conceptual similarities of three self-regulated learning inventories. British Journal of Educational Psychology, 77(1), 177-195. doi: 10.1348/000709905X90876

Muñiz, J., Elosua, P., \& Hambleton, R. K. (2013). Directrices para la traducción y adaptación de los tests $\left(2^{\mathrm{a}}\right.$ ed.). Psicothema, 25(2), 151-157. Recuperado de http://www.psicothema.com

Nunnally, J. C., \& Bernstein, I. (1995). Teoría psicométrica. Madrid, España: MCGraw-Hill.

Núñez J. L., Martín-Albo, J., \& Navarro, J. G. (2005). Validación de la versión española de la Échelle de Motivation en Éducation. Psicothema, 17(2), 344349. Recuperado de http://www.psicothema.com

Pardo, A., \& Ruiz, M. (2002). SPSS 11 Guía para el análisis de datos. Madrid:Mc Graw Hill.

Pintrich, P. R. (2000). The role of goal orientation in self-regulated learning. En M. Boekaerts, P. R. Pintrich, \& M. Zeidner (Eds.), Handbook of Self-regulation (pp. 451-502). San Diego, CA: Academic Press. doi: 10.1016/B978-012109890-2/50043-3

Pintrich, P. R., \& De Groot, E. V. (1990). Motivational and self regulated learning components of classroom academic performance. Journal of Educational Psychology, 82(1), 33-40. doi: 10.1037/0022-0663.82.1.33

Pintrich, P. R., Smith, D. A., García, T., \& McKeachie, W. (1991). A Manual for the use of the Motivated Strategies for Learning Questionnaire (MSLQ). Ann Arbor: University of Michigan. Recuperado de https://eric.ed.gov/?id=ED338122

Pintrich, P. R., Smith, D. A. F., García, T., \& McKeachie,W. J. (1993). Reliability and predictive validity of the Motivated Strategies for Learning Questionnaire (MSLQ). Educational and Psychological Measurement, 53(3), 801-813. doi: $10.1177 / 0013164493053003024$

Purdie, N., \& Hattie, J. (1996). Cultural differences in the use of strategies for self-regulated learning. American Educational Research Journal, 33(4), 845-871. Recuperado de http://www.jstor.org/ stable/1163418?seq=1\#page_scan_tab_contents

Ramirez-Dorantes, M. C., Canto y Rodríguez, J. E., BuenoAlvarez, J. A., \& Echazarreta-Moreno, A. (2013). Psychometric validation of the Motivated Strategies for Learning Questionnaire, with Mexican university students. Electronic Journal of Research in Educational Psychology, 11(1), 193-214. Recuperado de http://www.investigacion-psicopedagogica.org/ revista/new/index.php

Rao, N., \& Sachs, J. (1999). Confirmatory factor analysis of the Chinese version of the Motivated Strategies for Learning Questionnaire. Educational \& Psychological Measurement, 59(6), 1016-1029. doi: $10.1177 / 00131649921970206$

Rotgans, J. I., \& Schmidt, H. G. (2010). The Motivated Strategies for Learning Questionnaire: A measure for students' general motivational beliefs and learning strategies? The Asia-Pacific Education Researcher, 19(2), 357-369. doi: 10.3860/taper.v19i2.1603

Ryan, R. M., \& Deci, E. L. (2000). Intrinsic and extrinsic motivations: Classic definitions and new directions. Contemporary Educational Psychology, 25(1), 5467. doi: 10.1006/ceps.1999.1020

Schunk, D. H. (2005). Self-regulated learning: The educational legacy of Paul R. Pintrich. Educational Psychologist, 40(2), 85-94. doi: 10.1207/ s15326985ep4002_3

Vallerand, R. J., Blais, M. R., Brière, N. M., \& Pelletier, L. G. (1989). Construction et validation de l'Échelle de Motivation en Éducation (EME) [Construction and validation of the Motivation toward Education Scale]. Canadian Journal of Behavioural Sciencel Revue Canadienne des Sciences du Comportement, 21(3), 323-349. doi: 10.1037/h0079855

Van Widenfelt, B. M., Treffers, P. D. A., De Beurs, E., Siebelink, B. M., \& Koudijs, E. (2005). Translation and cross-cultural adaptation of assessment instruments used in psychological research with 
children and families. Clinical Child and Family Psychology Review, 8(2), 135-147. doi: 10.1007/ s10567-005-4752-1

Veenman, M. V. J. (2011). Alternative assessment of strategy use with self-report instruments: A discussion. Metacognition and Learning, 6(2), 205-211. doi: 10.1007/s11409-011-9080-x

Wolters, C. A. (2003). Regulation of motivation: Evaluating an underemphasized aspect of self-regulated learning. Educational Psychologist, 38(4), 189-205. doi: 10.1207/S15326985EP3804_1 